\title{
ASPECTS OF NON-EQUILBRIUM QUANTUM FIELD THEORY IN RELATIVISTIC HEAVY ION COLLISIONS
}

\author{
C. Greiner ${ }^{1}$, S. Juchem ${ }^{2}$ and Z. Xu ${ }^{1,2}$ \\ ${ }^{1}$ Institut für Theoretische Physik der Johann Wolfgang Goethe Universität, D-60054, Frankfurt \\ am Main, Germany \\ ${ }^{2}$ Institut für Theoretische Physik der Justus Liebig Universität, D-35392, Giessen, Germany
}

\begin{abstract}
In this lecture we review recent progress in various aspects of non-equilibrium QFT with respect to relativistic heavy ion collisions. As a first and rather general study we summarize our (numerical) investigations for a dissipative quantum time evolution of $\phi^{4}$-field theory for a spatially homogeneous system in $2+1$ space-time dimensions on the basis of the Kadanoff-Baym equations. The initial conditions can be chosen arbitrarily and far from equilibrium. The calculations demonstrate how thermalization and chemical equilibration is achieved ab initio from the underlying QFT. As a possible candidate for a non-equilibrium phase transition phenomena we then adress the stochastic formation of disoriented chiral condensates. Finally we elaborate on a new $3+1$ dimensional Monte Carlo parton cascade solving kinetic Boltzmann processes including inelastic multiplication processes $(g g \leftrightarrow g g g)$ in an unified manner, where the back reaction channel is treated for the first time fully consistently.
\end{abstract}

\section{Introduction, overview and summary}

The prime intention for present ultrarelativistic heavy ion collisions at CERN and at Brookhaven lies in the possible experimental identification of the quark gluon plasma (QGP). The evolving system in the reaction is at least initially far from any (quasi-)equilibrium configuration. Not only for this reason, nonequilibrium quantum field theory has become a major topic of research for describing transport processes in various areas of physics like in cosmological particle physics as well as condensed matter physics. The multidisciplinary aspect arises due to a common interest to understand the various real-time relaxation phenomena of quantum dissipative systems.

A seminal work in solving numerically for the first time the so called KadanoffBaym equations of evoltion equations for out-of-equilibrium one-particle Green's 
function has been carried out by Danielewicz [1], who investigated a spatially homogeneous system with a deformed Fermi sphere in momentum space for the initial distribution of occupied momentum states in order to model the initial condition of a heavy-ion collision in the nonrelativistic domain. In comparison to a standard on-shell semi-classical Boltzmann equation the solutions showed quantitative differences like a larger collective relaxation time for achieving complete kinetic equilibration. Similar quantum modifications in the equilibration and momentum relaxation have been found for a relativistic situation in [2], where also a faster equilibration can occur at higher bombarding energies.

In section 2 we summarize our findings of a recent and very detailed study concerning the quantum time evolution of $\phi^{4}$-field theory for a spatially homogeneous system in $2+1$ space-time dimensions by including the mean-field (tadpole) and collisional (sunset) self-energies [3]. As an important and new step in the field, we point out the dynamics of the spectral ('off-shell') distributions of the excited quantum modes and the different phases in the approach to equilibrium described by Kubo-Martin-Schwinger (KMS) relations for full thermal equilibrium states, the latter being the only translation invariant solution and representing the stationary fixed points of the dissipative coupled equation of motions. Concentrating further on the importance of off-shell effects, far off-shell $1 \leftrightarrow 3$ decay processes are responsible for chemical equilibration. The physical process corresponds to a consistent treatment of Bremsstrahlung type processes in the medium by the interplay of production, propagation and subsequent decay of highly virtual mode excitations.

As an exotic candidate for a direct signature stemming from the QGP phase transition (and which can be adressed in RHIC experiments at Brookhaven) we summarize in section 3 ideas of stochastic formation of so called disoriented chiral condensates (DCC). The idea of DCC [4] first appeared in a work of Anselm but it was made widely known due to Bjorken, and Rajagopal and Wilczek. The spontaneous growth and subsequent decay of these configurations emerging after a rapid chiral phase transition from the QGP to the hadronic world would give rise to large collective fluctuations in the number of produced low momentum neutral pions compared to charged pions. Our work concentrates on the important question on the likelihood of an instability leading potentially to a large DCC yield of low momentum pions [5]. It reveals that an experimentally feasible DCC, if it does exist in nature, has to be a rare event with some finite probability following a nontrivial and nonpoissonian distribution on an event by event basis. DCCs could then (only) be revealed experimentally by inspecting higher order factorial cumulants $\theta_{m}(m \geq 3)$ in the sampled distribution of low momentum pions.

In section 4 we finally detail on a new parton cascade scheme treating elastic and inelastic multiplication collisions in an unified manner [6]. For the first time the back reation channel $(g g g \rightarrow g g$ ) is treated fully consistently by re- 

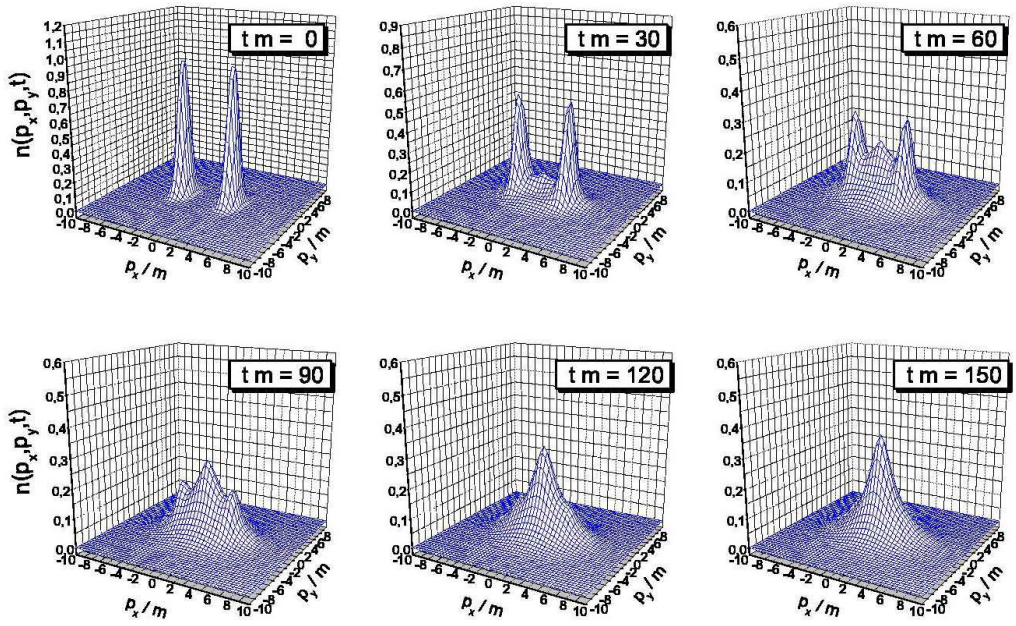

Figure 1. Characteristic evolution of the occupation number in momentum space within the full Kadanoff-Baym dynamics starting from an initially non-isotropic shape. The occupation number correspondes directly to the equal-time Green function via the relation 3 and is displayed for various times $\bar{t} \cdot m=0,30,60,90,120,150$.

specting detailed balance within the same algoritm. With this we can adress the important question of thermalization and early pressure build up on a partonic level for heavy ion collisions at RHIC. First (and preliminary) results show that indeed the gluon multiplication via Bremsstrahlung (and absorption) is of utmost importance, where thermalization is achievd on a timescale of about 1 $\mathrm{fm} / \mathrm{c}$.

\section{Dissipative quantum dynamics for $\phi^{4}$-theory}

The starting point of our recent numerical investigation are the causal DysonSchwinger equations for certain real-time one-particle Green's functions, which are also called Kadanoff-Baym equations [3]:

$$
\begin{aligned}
-\left[\partial_{\mu}^{x} \partial_{x}^{\mu}+m^{2}\right] & G^{>/<}(x, y)=\Sigma^{\delta}(x) G^{>/<}(x, y) \\
+\int_{t_{0}}^{x_{0}} d z_{0} \int d^{d} z & {\left[\Sigma^{>}(x, z)-\Sigma^{<}(x, z)\right] G^{>/<}(z, y) } \\
-\int_{t_{0}}^{y_{0}} d z_{0} \int d^{d} z & \Sigma^{>/<}(x, z)\left[G^{>}(z, y)-G^{<}(z, y)\right]
\end{aligned}
$$


Besides the Hartree term, to next order in the coupling constant the non-local sunset self-energy enters as

$$
\Sigma^{>/<}(x, y)=-\frac{\lambda^{2}}{6}\left[G^{>/<}(x, y)\right]^{3} .
$$

With this the Kadanoff-Baym equations are in closed form and can thus be (numerically) integrated. In the following we summarize some major findings of such an analysis for a $2+1$ dimensional and spatially homogenous system being prepared by some non-equilibrium initial configuration of excited modes in momentum space at $t=0$ via

$$
2 \omega_{\mathbf{p}} i G_{\phi \phi}^{<}(\mathbf{p}, t, t)=2 n(\mathbf{p}, t)+1 .
$$

A typical evolution for this Green's function is depicted in fig. 1. Starting from an initially far non-isotropic shape it develops towards a rotational symmetric distribution in momentum space. Initially there is no occupation along the $p_{y^{-}}$ momentum axis. As a function of time this area of momentum space is getting filled which takes about 100 time units. In fig. 2 the occupation number spectra along the transverse momentum axis is depicted. A quasi exponetial slope is experienced on the very onset of the ongoing evolution, where the system is still far from kinetic equilibrium, as most energy is still stored in excitations along the longitudinal axis. Hence, a 'thermal' spectra along the transverse direction as given by the present example does not necessarily imply that the (complex) system is close to kinetic and thermal equilibrium. (This could be also true for relativistic heavy ion collisions - see eg [7] - where the transverse spectra typically look 'thermal'.)

On the other hand, we observe that starting from very different initial conditions for systems containing the same energy that the single momentum modes converge to the same respective numbers for large times as characteristic for a system in equilibrium. The different momentum modes in Fig. 3 typically show a three-phase structure. For small times $(t \cdot m<10)$ one finds damped oscillations that can be identified with a typical switching on effect at $t=0$, where correlations build up and dephasing of the various modes does occur. For 'intermediate' time scales $(10<t \cdot m<100)$ one observes a strong change of all momentum modes in the direction of the final stationary state. We address this phase to 'kinetic' equilibration and point out, that the momentum modes can temporarily even exceed their respective equilibrium value (see the evolution of the distribution D1). We remark that this behaviour of 'overshooting' as in the particular case of D1 - is not observed in a simulation with a kinetic Boltzmann equation. Hence this highly nonlinear effect must be attributed to quantal off-shell and memory effects not included in the standard Boltzmann limit. The third phase, i.e. the 'late' time evolution is characterized by a smooth 


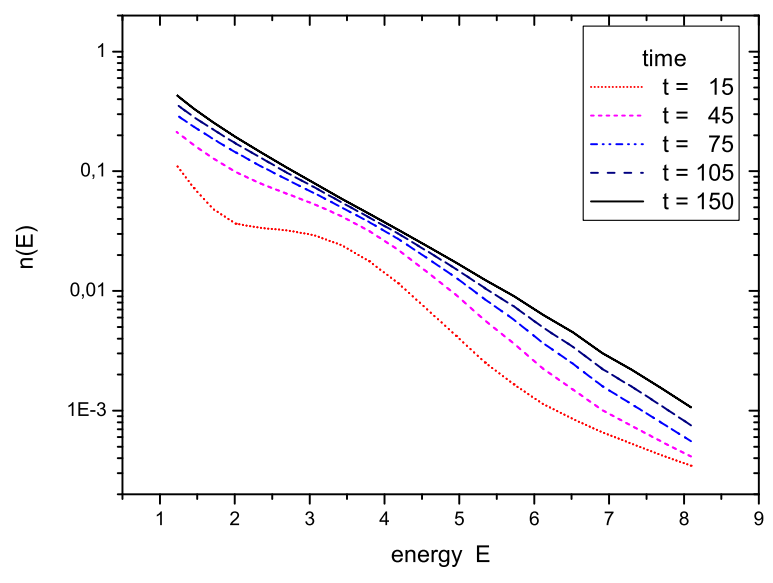

Figure 2. Occupation number spectra along the transverse momentum axis $\left(p_{x}=0, p_{y}\right)$ plotted versus (transverse) energy. The time is taken in units of the inverse mass $m$, the energy is given in units of $\mathrm{m}$.

approach of the single momentum modes to their respective equilibrium values. As we will now explain, this phase is adequately characterized by chemical processes changing the 'number' of (quasi-)particles or excitations. (It turns out that this rate for chemical equilibration is only slightly smaller than that of kinetic equilibration for the coupling parameter chosen [3].)

In principle one can now study the time evolution of the spectral function as a function of time. This is an important and lively topic, but we refer the interested reader to the paper [3]. In Fig. 4 (lower part) the spectral function $A\left(\mathbf{p}, p_{0}, \bar{t}\right)$ for the initial distribution D2 at very late times for various momentum modes is depicted as a function of the energy $p_{0}$. The system has nearly equilibrated and the spectral function are rather broad, reflecting a strongly interacting system via the choice of a rather large coupling constant.

At perfect thermal equilibrium the famous KMS condition of the Green functions hold dictating that the equilibrium form of the complete and off-shell distribution function at temperature $T$ is simply given via

$$
N_{e q}\left(\mathbf{p}, p_{0}\right)=N_{e q}\left(p_{0}\right)=\frac{1}{\exp \left(p_{0} / T\right)-1}=N_{\text {bose }}\left(p_{0} / T\right)
$$

which is the well-known Bose distribution. The distribution function $N\left(p_{0}\right)$ as extracted from the calculation is displayed in Fig. 4 (upper part) for the same momentum modes as a function of the energy $p_{0}$. We find that $N\left(p_{0}\right)$ for 


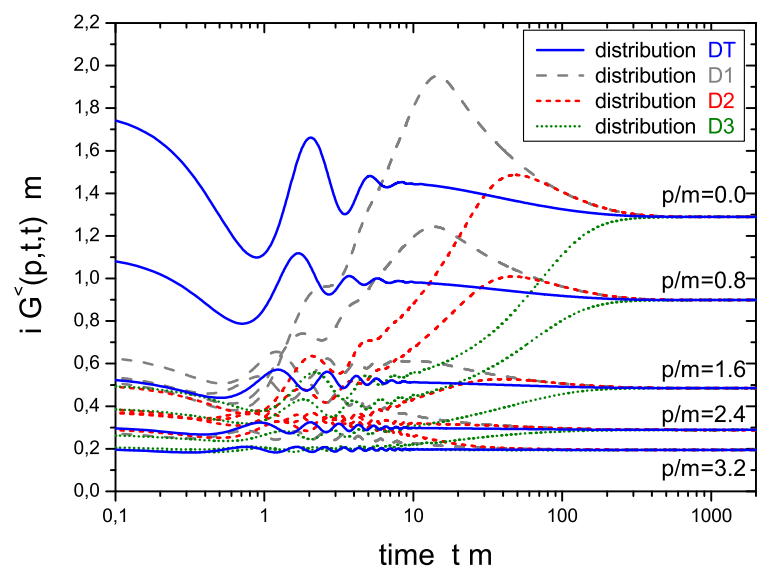

Figure 3. Time evolution of selected momentum modes of the equal-time Green function $|\mathbf{p}| / m=0.0,0.8,1.6,2.4,3.2$ (from top to bottom) for four different initial configurations D1, D2, D3 and DT (characterized by the different line types) with the same energy density. All momentum modes assume the same respective equilibrium value for long times $(t \cdot m>500)$ independent of the initial state.

all individual momentum modes can be fitted by a single Bose function with temperature $T / m=1.835$. Thus the distribution function emerging from the Kadanoff-Baym time evolution for $t \rightarrow \infty$ approaches indeed a Bose function in the energy $p_{0}$ that is independent of the momentum as demanded by the equilibrium form. The KMS conditions are approached as a dynamical fixed point in the complex non-equilibrium evolution.

We note that the chemical potential $\mu$ - used as a second fit parameter - is indeed close to zero for these late times as expected for the correct equilibrium state of the neutral $\phi^{4}$-theory which is characterized by a vanishing chemical potential $\mu$ in equilibrium. This is a consequence of the exact treatment. A kinetic Boltzmann equation in general would lead to a stationary state for $t \rightarrow \infty$ with a finite chemical potential.

In Fig. 5 the width $\Gamma$ is displayed as a function of the energy $p_{0}$. The width shows two maxima for all momentum modes. The first is characterized by an increase towards a maximum around the on-shell energy and falling off beyond. This behaviour stems from the $2 \leftrightarrow 2$ processes in the self-energies. Particles can be scattered by other particles - present in the system at finite temperature - such that they achieve the shown (collisional) damping width. On the other hand, particles with sufficient energy can decay into three other 


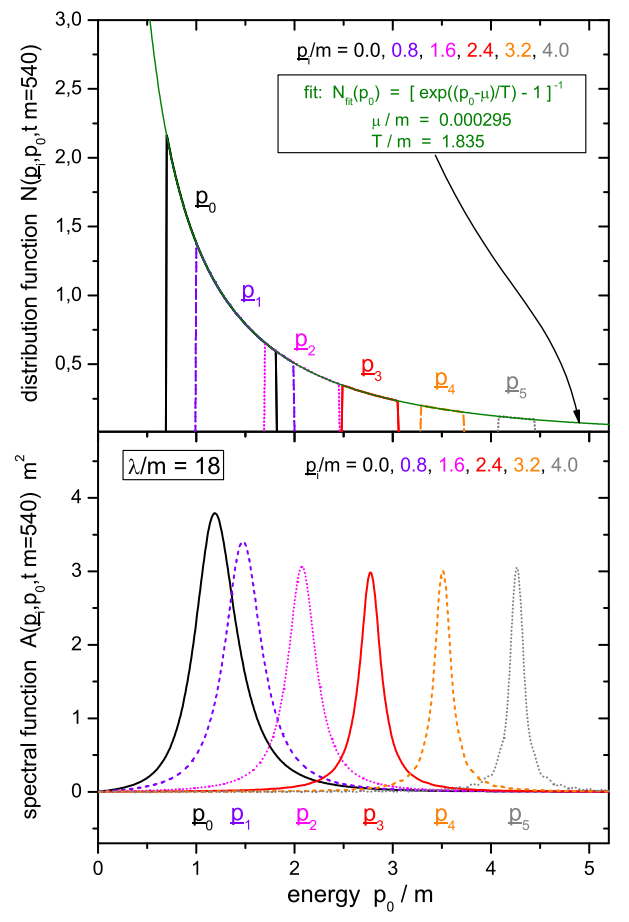

Figure 4. Spectral function $A$ for various momentum modes $|\mathbf{p}| / m=0.0,0.8,1.6,2.4,3.2$, 4.0 as a function of energy for late times $\bar{t} \cdot m=540$ (lower part). Corresponding distribution function $N$ at the same time for the same momentum modes (upper part). All momentum modes with respective off-shell equilibration can be fitted with a single Bose function of temperature $T_{e q} / m=1.835$ and a chemical potential close to zero.

particles. Above (approximately) the threshold of $p_{0, t h}(\mathbf{p})=\sqrt{\mathbf{p}^{2}+\left(3 m^{*}\right)^{2}}$ these $1 \leftrightarrow 3$ processes lead to an increase of the width (as marked for the highest momentum mode by the second thin line). The inclusion of this part of the particle width in the spectral function is responsible for the process of chemical equilibration. Within the Kadanoff-Baym scheme the off-shell $1 \leftrightarrow 3$ transitions lead to a violation of 'particle number'. The physical interpretation of the dynamics is a consistent treatment of Bremsstrahlungs processes in the medium via the interplay of production, propagation and subsequent decay of highly virtual mode excitations. 


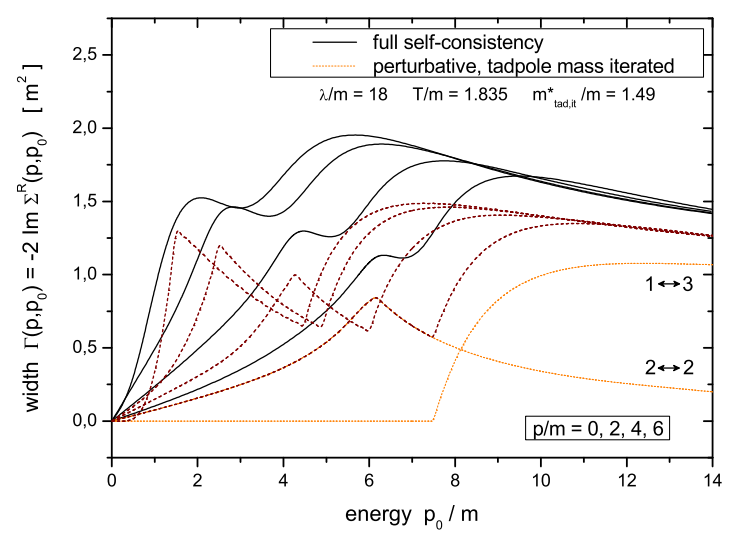

Figure 5. Self-consistent width (solid lines) and perturbative width (dashed lines) as a function of the energy $p_{0} / m$ for various momentum modes $|\mathbf{p}| / m=0,2,4,6$ for a thermal system. For the highest momentum mode $|\mathbf{p}| / m=6$ of the perturbative calculation, the collision contribution $(2 \leftrightarrow 2)$ and the decay contribution $(1 \leftrightarrow 3)$ to the width are explicitly displayed.

\section{Stochastic disoriented chiral condensates}

In this section we give a brief report on our findings on the stochastic nature of DCC formation and how to possibly identify their existence experimentally[5]. This work resulted from an investigation[8], where we adressed the potential likeliness of an instability leading to a sufficiently large DCC event during the evolution of a fireball undergoing a phase transition within the linear $\sigma$-model.

The main idea is that the final fluctuations depend critically on the initial conditions chosen for the evolving chiral order parameter, thus deciding to some extent whether the system enters temporarily the instable region during the 'roll-down' period of the order parameter[8, 5]. In fact, a semi-classical and dissipative dynamics of the order parameter and the pionic fields can be obtained by an effective and complex action, where the interaction with the thermal pions has been integrated out. To the end, we have utilized the following stochastic Langevin equations of motion for the order parameters $\Phi_{a}=\frac{1}{V} \int d^{3} x \phi_{a}(\mathbf{x}, t)$ in a D-dimensional ('Hubble') expanding volume $V(\tau)$ to describe the evolution of collective pion and sigma fields $[8,5]$ :

$$
\begin{aligned}
\ddot{\Phi_{0}}+\left(\frac{D}{\tau}+\eta\right) \dot{\Phi}_{0}+m_{T}^{2} \Phi_{0} & =f_{\pi} m_{\pi}^{2}+\xi_{0}, \\
\ddot{\Phi}_{i}+\left(\frac{D}{\tau}+\eta\right) \dot{\Phi}_{i}+m_{T}^{2} \Phi_{i} & =\xi_{i},
\end{aligned}
$$




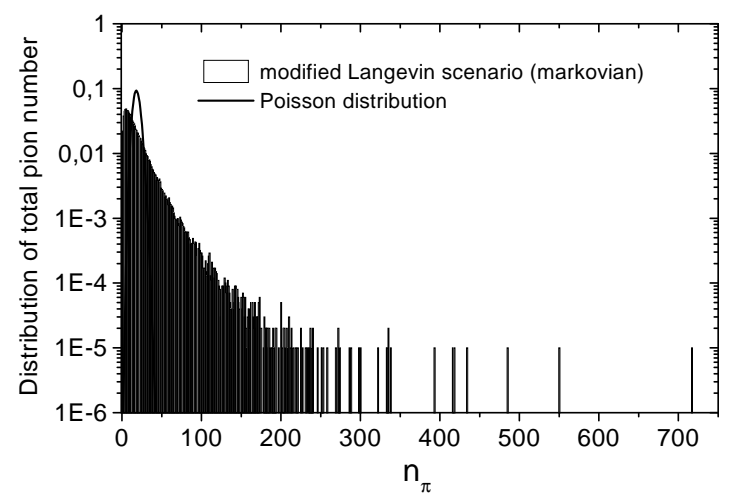

Figure 6. Statistical distribution $P\left(n_{\pi}\right)$ of the final yield $n_{\pi}$ in low momentum pion number of a single DCC for a rapidly expanding situation (see ref.[5] for details) compared with a corresponding simple poissonian distribution.

with $\Phi_{0}=\sigma$ and $\Phi_{i}=\left(\pi_{1}, \pi_{2}, \pi_{3}\right)$ being the chiral meson fields and $m_{T}^{2}=$ $\lambda\left(\Phi_{0}^{2}+\sum_{i} \Phi_{i}^{2}+\frac{1}{2} T^{2}-f_{\pi}^{2}\right)+m_{\pi}^{2}$ denotes the effective transversal ('pionic') masses. These coupled Langevin equations resemble in its structure a phenomenological Ginzburg-Landau description of phase transition. Aside from a theoretical justification one can regard the Langevin equation as a practical tool to study the effect of thermalization on a subsystem, to sample a large set of possible trajectories in the evolution, and to address also the question of all thermodynamically possible initial configurations in a systematic manner.

In fig. 6 we show the statistical distribution in the number of produced long wavelength pions $N_{\pi}$ out of the evolving chiral order fields within the DCC domain $V(\tau)$ for one particular set of parameters[5]. A rather rapid and $(D=) 3$ dimensional expansion has been employed. (The results majorly depend on how fast the assumed cooling and expansion proceeds.) In general one finds that only for $\mathrm{D}=3$ and sufficiently fast expansion individual unusual strong fluctuations of the order of 50-200 pions might occur, although the average number $\left\langle n_{\pi}\right\rangle$ of the emerging long wavelength pions only posesses a moderate and undetectable value of $5-20$.

In these interesting cases the final distribution does not follow a usual Poissonian distribution (comp. fig. 6), which represents a very important outcome. (Critical, dynamical) Fluctuations with a large number of produced pions are still likely with some small but finite probability! Unusual events out of sample contain a multiple in the number of pions compared to the average. One can interpret those particular events as semi-classical 'pion bursts' similar to the mystique Centauro candidates. If DCCs are being produced, an experimental 


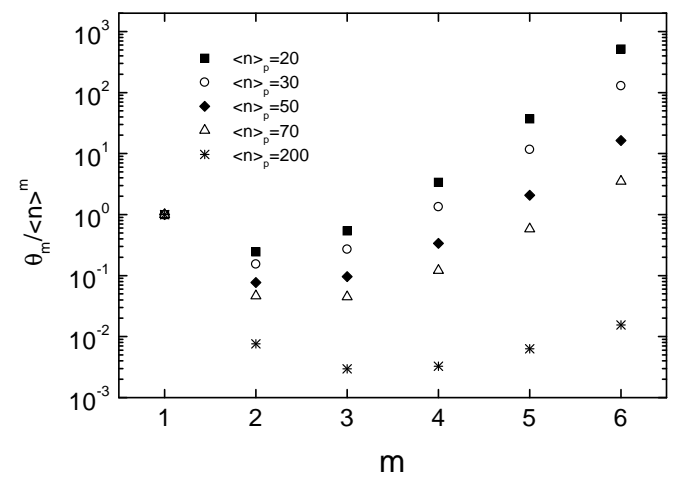

Figure 7. The reduced factorial cumulants for $m=1$ to 6 for the pion number distribution of low momentum stemming from a single emerging DCC (of the previous figure) and an additional poissonian distributed background pion source with different mean values $\langle n\rangle_{P}=20-200$.

finding will be a rare event following a strikingly, nontrivial and nonpoissonian distribution. A dedicated event-by-event analysis for the experimental programs (e.g. the STAR TPC at RHIC) is then unalterable.

The further analysis of this unusual distribution by means of the cumulant expansion shows that the reduced higher order factorial cumulants

$\theta_{m} /<n_{\pi}>^{m}$ for $m \geq 3$ exhibit an abnormal, exponentially increasing tendency, as illustrated in fig. 7. There an additional incoherent Poissonian background of (low momentum) pions stemming from other possible sources has been added. We advocate that an analysis by means of the higher order cumulants serves as a powerful signature. In conclusion, the occurence of a rapid chiral phase transition (and thus DCCs) can be identified experimentally by inspecting higher order facorial cumulants $\theta_{m}(m \geq 3)$ for taken distributions of low momentum pions.

\section{Elastic and inelastic multi-particle collisions in a parton cascade}

A major goal of the experiments at RHIC and LHC is the creation and observation of the quark-gluon-plasma. To describe the dynamics of ultrarelativistic heavy ion collisions (uRHICs), and to address the crucial question of thermalization and early pressure build up, we have developed a kinetic parton cascade algorithm [6] inspired by perturbative QCD including inelastic ('Bremsstrahlung') collisions $g g \leftrightarrow g g g$ besides elastic collisions. It is the aim to get a more detailed and quantitative understanding of the early dynamical 
stages of deconfined matter and to test various initial conditions for the liberated gluons, than envoking ad hoc varoius phenomenological, hydrodynamical ansaetze. An parton cascade analysis incorporating only binary $2 \leftrightarrow 2$ collisions described via direct $\mathrm{pQCD}$ scattering processes shows that thermalization and early quasi-hydrodynamic behaviour (for achieving sufficient elliptic flow) can not be buildt up or maintained, but only if a much higher cross section is being employed [9]. The possible importance of the inelastic reactions was indeed raised in the so called 'bottom up thermalization' picture [10]. It is intuitively clear that gluon multiplication should lead to a faster equilibration.
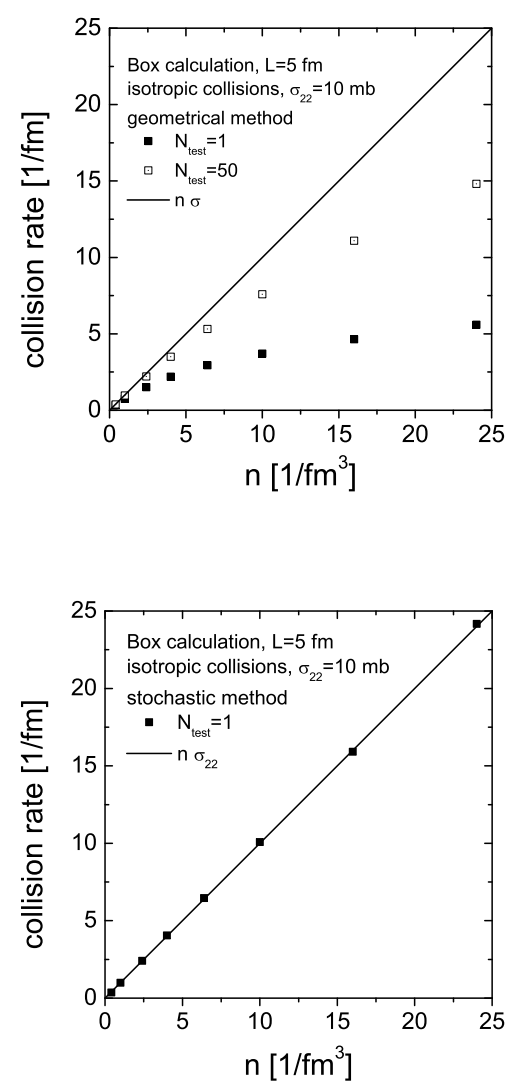

Figure 8. Collision rates in the simulation versus the ideal rates $1 /(n \sigma)$. The total cross section is fixed to $10 \mathrm{mb}$. In the upper (lower) figure the geometrical (stochastic) method is employed.

In developing the algorithm special emphasis is put on obeying the principle of detailed balance among the gain and loss contributions. The standard 
incorporation of $2 \leftrightarrow 2$ scattering processes in a transport description is based on the geometric interpretation of the cross section [11]. For large particle densities, however, such an implementation leads to considerable problems to generate a causal collision sequence among the various partons, leading to a severe reduction of the collision rate compared to the one dictated by the Boltzmann equation, see fig. 8 . In principle, this can be cured by the test particle method. However, for such a situation the stochastic method (see eg [12]) is better suited to solve the Boltzmann equation directly via transition rates in sufficiently small spatial cells, see fig. 8. Here the actual collision rate being realized in the simulation fully equals the ideal one of the Boltzmann equation which shall be simulated. One can go to arbitrary high density. Moreover, this method can, in principle, be generalized to (any) multi-particle scattering processes.

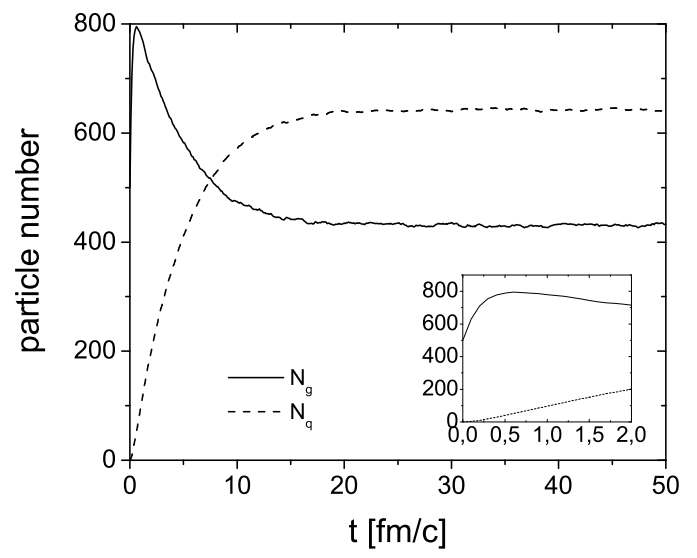

Figure 9. Time evolution of the gluon and quark numbers for the box calculation. The gluon number starts at 500 .

To demonstrate the applicability of the method, we show in the following the formation of a quark gluon plasma within a fixed box and study the way of kinetic and chemical equilibration for different parton species. The initial conditions of the partons entering the cascade are given by the multiple minijets (partons with $p_{t}>2 \mathrm{GeV} / \mathrm{c}$ ) production [13] from the binary nucleon-nucleonscattering in a nucleus-nucleus-collision according to the differential jet cross section:

$$
\frac{d \sigma_{j e t}}{d p_{T}^{2} d y_{1} d y_{2}}=K \sum_{a, b} x_{1} f_{a}\left(x_{1}, p_{T}^{2}\right) x_{2} f_{b}\left(x_{2}, p_{T}^{2}\right) \frac{d \sigma_{a b}}{d \hat{t}}
$$


where $p_{T}$ is the transverse momentum and $y_{1}$ and $y_{2}$ are the rapidities of the produced partons. The minijets are considered in the central rapidity interval $(-0.5: 0.5)$ at RHIC energy of $\sqrt{s}=200 \mathrm{GeV}$. The initial partons are distributed uniformly in a cubic box of size $3 \mathrm{fm}$, which approximately corresponds to the central region of heavy ion collions at some early initial time.

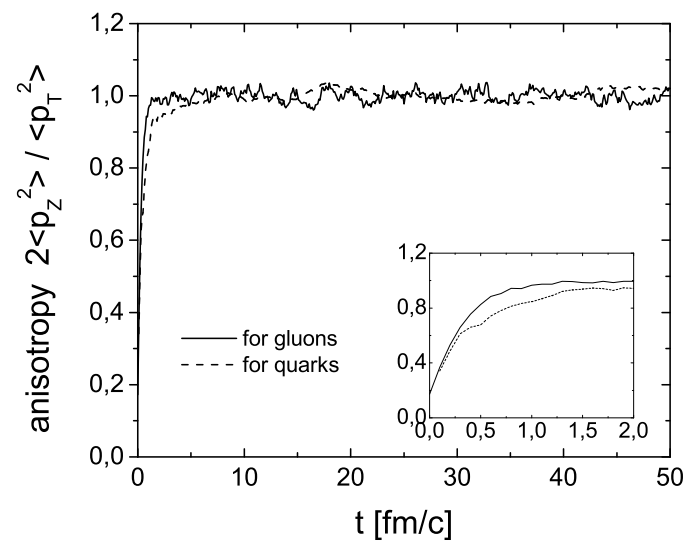

Figure 10. Time evolution of the momentum anisotropy for the QGP in a box.

We consider here gluon and quarks with two flavors as parton species. Collision processes are the two-body elementary parton-parton scatterings and three-body process $g g \leftrightarrow g g g$ in leading-order of pQCD, employing effective Landau-Pomeranchuk-Migdal suppression and standard screening masses for the infrared sector of the scattering amplitude.

Fig. 9 shows the time evolution of the gluon and quark number. Gluon equilibration undergoes two stages: at first the gluon number increases rapidly and then smoothly evolves to its final equilibrium value together with the quark number at a much lower rate. The early stage of gluon production also leads to an immediate kinetic equilibration of the momentum distribution (see fig. 10 and fig. 12) as well as to a rather abrupt lowering of the temperature by soft gluon emission until detailed balance among gain and loss contributions is reached (see fig. 11).

The later slower evolution is then governed by chemical equilibration of the quark degrees of freedom. The final temperature of fig. 11 is identical to the late slope parameter of the energy spectra depicted in fig. 12.

With the present cascade we are aiming to study in detail real HIC incorporating various initial conditions like minijets or the color glass condensate. In 


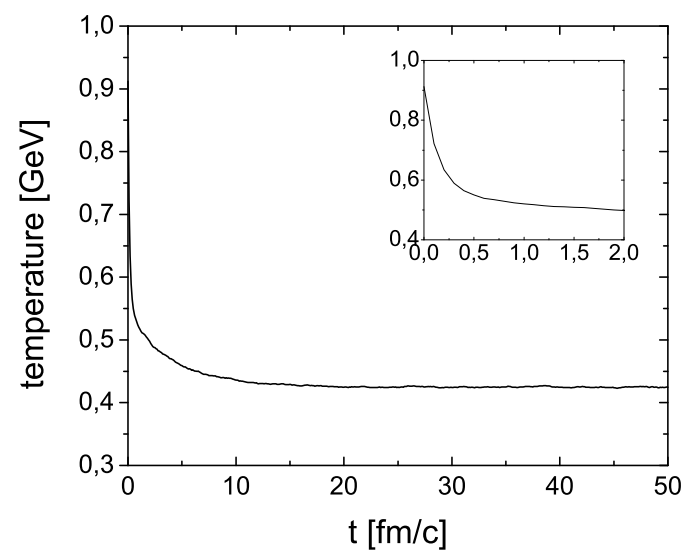

Figure 11. Time evolution of the effective temperature $T=\frac{E}{3 N}$.

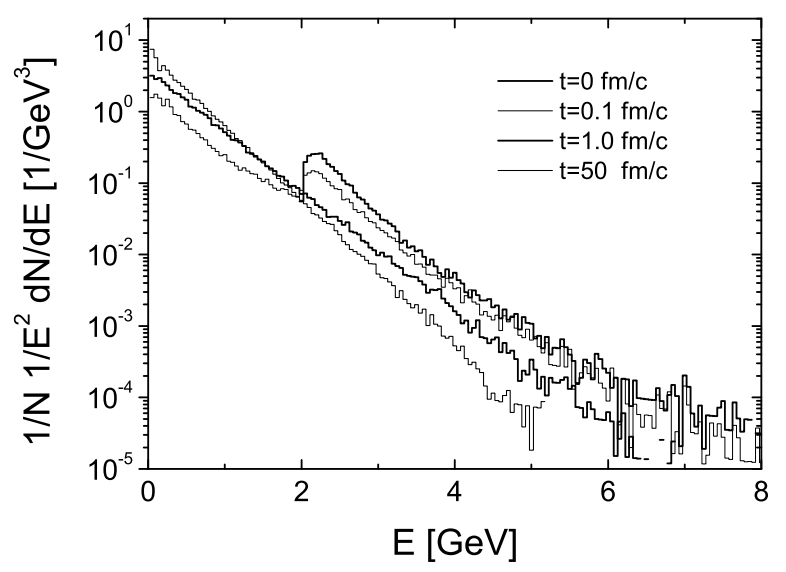

Figure 12. Occupation versus energy spectra at different times. within the box calculation. At $t=0$ only minijets with $E>2 \mathrm{GeV}$ are populated. Energy degradation to lower momenta proceeds rapidly by gluon emission.

fig. 13 we depict one such first (and still preliminary, as yet not fully tested) calculation with minijet initial conditions. Thermalization and chemical equilibration as propoesed in the bottom up picture [10] can then thoroghly be tested 


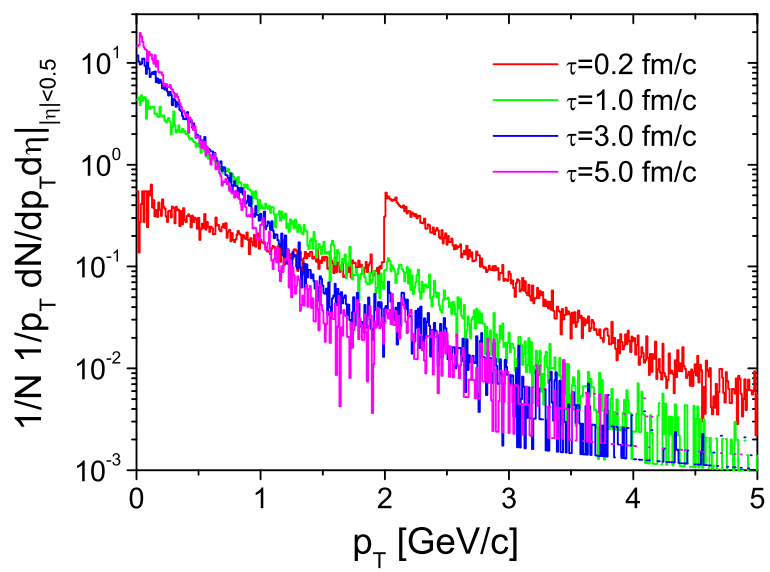

Figure 13. Transverse momentum spectrum at midrapidity at different times for a real, central fully 3-D (including transversal expansion) ultrarelativistic heavy ion collision. Only the partons residing in a cylinder of radius $R \leq 5 \mathrm{fm}$ are taken for the plot. The initial out-of-equilibrium conditions are given by minijets and are distributed via the corresponding overlap function in space-time. The calculation is preliminary. From $t=0$ on first only minijets with $E>2 \mathrm{GeV}$ are populated. Energy degradation to lower momenta proceeds rapidly by gluon emission within the first fm/c. Maintenance of (quasi-)kinetic and chemical equilibrium is given up to $4 \mathrm{fm} / \mathrm{c}$, where longitudinal and transversal (quasi-hydrodynamical) work is done resulting in a continous lowering of the temperature. On the other hand the initial non-equilibrium high momentum tail following a standard power-law for mini-jet production is partly surviving.

within this approach. Furthermore we will study the impact parameter dependence of the transverse energy in order to understand elliptic and transverse flow at RHIC within this new scheme of a kinetic parton cascade including inelastic interactions by comparing to experimental data.

\section{Acknowledgments}

The work refered to in section 2 has been done together with W. Cassing and the collaboration is gratefully acknowledged. The various work has been supported by BMBF, DFG and GSI Darmstadt.

\section{References}

[1] P. Danielewicz, Ann. Phys. (N.Y.) 152 (1984) 305.

[2] C. Greiner, K. Wagner, and P.-G. Reinhard, Phys. Rev. C 49, 1693 (1994). 
[3] S. Juchem, W. Cassing and C. Greiner, 'Quantum dynamics and thermalization for out of equilibrium $\phi^{4}$ theory', arXiv:hep-ph/037353, Physical Review D69 (2004) in press.

[4] D. Anselm, Phys. Lett. B 217, 169 (1989); J.D. Bjorken, Int. J. Mod. Phys. A 7, 4819 (1992); K. Rajagopal and F. Wilczek, Nucl. Phys. B 404, 577 (1993).

[5] Z. Xu and C. Greiner, Phys. Rev. D 62, 036012 (2000).

[6] Z. Xu and C. Greiner, 'Elastic and inelastic multiplication collisions in a parton cascade treated in an unified manner', publication in preparation.

[7] B. Schenke and C. Greiner, 'Statistical description with anisotropic momentum distributions for hadron production in nucleus-nucleus collisions', arXiv:nucl-th/0305008

[8] T.S. Biró and C. Greiner, Phys. Rev. Lett. 79, 3138 (1997).

[9] D. Molnar and M. Gyulassy, Nucl. Phys. A 697, 495 (2002).

[10] R. Baier, A.H. Mueller, D. Schiff and D.T. Son, Phys. Lett. B 502, 51 (2001).

[11] D. Molnar and M. Gyulassy, Phys. Rev. C 62, 054907 (2000).

[12] A. Lang et al, J.Comp.Phys. 106 (1993), 391 (1993).

[13] K.J. Eskola et al., Nucl. Phys. B 323, 37 (1989); X.-N. Wang, Phys. Rep. 280, 287 (1997). 